\title{
Association between knee alignment and knee pain in patients surgically treated for medial knee osteoarthritis by high tibial osteotomy. A one year follow-up study
} Annette W-Dahl*1, Sören Toksvig-Larsen ${ }^{2}$ and Ewa M Roos ${ }^{3}$

\author{
Address: ${ }^{1}$ Department of Orthopedics, Clinical Sciences Lund, Lund University, Sweden, ${ }^{2}$ Department of Orthopedics, Hässleholm Hospital, \\ Hässleholm, Sweden and ${ }^{3}$ Institute of Sports Science and Clinical Biomechanics, University of Southern Denmark, Odense, Denmark \\ Email: Annette W-Dahl* - annette.w-dahl@med.lu.se; Sören Toksvig-Larsen - soren.toksvig-larsen@med.lu.se; \\ Ewa M Roos - eroos@health.sdu.dk \\ * Corresponding author
}

Published: 8 December 2009

BMC Musculoskeletal Disorders 2009, 10:154 doi:10.1186/1471-2474-10-154

This article is available from: http://www.biomedcentral.com/I47I-2474/I0/I54

(c) 2009 W-Dahl et al; licensee BioMed Central Ltd.

This is an Open Access article distributed under the terms of the Creative Commons Attribution License (http://creativecommons.org/licenses/by/2.0), which permits unrestricted use, distribution, and reproduction in any medium, provided the original work is properly cited.
Received: 15 June 2009

Accepted: 8 December 2009

\begin{abstract}
Background: The association between knee alignment and knee pain in knee osteoarthritis (OA) is unclear. High tibial osteotomy, a treatment option in knee OA, alters load from the affected to the unaffected compartment of the knee by correcting malalignment. This surgical procedure thus offers the possibility to study the cross-sectional and longitudinal association of alignment to pain. The aims were to study I) the preoperative association of knee alignment to preoperative knee pain and 2) the association of change in knee alignment with surgery to change in knee pain over time in patients operated on for knee OA by high tibial osteotomy.
\end{abstract}

Methods: I 82 patients (68\% men) mean age 53 years (34 - 69) with varus alignment having tibial osteotomy by the hemicallotasis technique for medial knee OA were consecutively included. Knee alignment was assessed by the Hip-Knee-Ankle (HKA) angle from radiographs including the hip and ankle joints. Knee pain was measured by the subscale pain ( $0-100$, worst to best scale) of the Knee injury and Osteoarthritis Outcome Score (KOOS) preoperatively and at one year follow-up. To estimate the association between knee alignment and knee pain multivariate regression analyses were used.

Results: Mean preoperative varus alignment was 170 degrees (153 - 178) and mean preoperative KOOS pain was 42 points $(3-86)$. There was no association between preoperative varus alignment and preoperative KOOS pain, crude analysis 0.02 points $(95 \% \mathrm{Cl}-0.6-0.7)$ change in pain with every degree of HKA angle, adjusted analysis 0.3 points $(95 \% \mathrm{Cl}-1.3-0.6)$.

The mean postoperative knee alignment was 184 degrees (I7I - 185). The mean change in knee alignment was 13 degrees $(0-30)$. The mean change in KOOS pain was $32(-16-83)$. There was neither any association between change in knee alignment and change in KOOS pain over time, crude analysis 0.3 point $(95 \% \mathrm{Cl}-0.6-1.2)$, adjusted analysis 0.4 points $(95 \% \mathrm{Cl} 0.6-1.4)$.

Conclusion: We found no association between knee alignment and knee pain in patients with knee $O A$ indicating that alignment and pain are separate entities, and that the degree of preoperative malalignment is not a predictor of knee pain after high tibial osteotomy. 


\section{Background}

Varus and valgus malalignment are associated with medial and lateral knee osteoarthritis (OA) respectively. In natural history cohorts of knee OA, severity of malalignment has been shown to be associated with pain severity $[1,2]$. Additionally, frequent knee symptoms (i.e. pain, aching or stiffness on most days of the past month) was found to increase with increasing varus malalignment over 15 month [3]. In other studies malalignment was not associated with pain [4-6]. The relation of knee alignment and knee pain is thus still unclear and to our knowledge the association of alignment and pain has not previously been assessed in patients undergoing an intervention changing malalignment.

High tibial osteotomy (HTO) is a disease modifying intervention that reduces the tibiofemoral load in the damaged compartment of the knee joint. The purpose of HTO is to decrease malalignment, reduce pain, enhance function as well as delay or avoid the need of knee arthroplasty in younger and/or physically active patients with uni-compartmental knee OA. HTO offers the possibility to study the cross-sectional and longitudinal relation of knee alignment to knee pain.

Our aims were to study 1) the preoperative association of knee alignment determined as the Hip-Knee-Ankle (HKA) angle to preoperative knee pain and 2) the association of change in knee alignment with surgery to change in knee pain preoperatively compared to at one year postoperatively in patients operated on for knee OA by high tibial osteotomy using the hemicallotasis technique (HCO).

\section{Methods \\ Patients}

182 patients ( $68 \%$ men) mean age 53 year (range 34 - 69) scheduled for high tibial osteotomy (HTO) for medial knee OA, were consecutively included. The indication of surgery by the HCO is a consideration based on several aspects, as the presence of radiographic unicompartmental knee OA, knee alignment, pain, disability and level of activity both in working life and leisure time. When the orthopedic surgeon, in the present study one surgeon (STL) assessed all subjects, found an indication for HCO, the patient was given written and verbal information in a special outpatient clinic for patients treated by external fixation and the final decision on surgery was taken.

Of the 182 patients, 156 patients (86\%) were available at the one-year follow-up. Fourteen patients did not return the questionnaire, two patients were revised to a total knee replacement, two patients had other surgeries, one patient had surgery in the contra lateral knee at time to follow-up and one patient had died.

\section{Radiographic assessment and classification of $O A$}

Standing anteroposterior images of the knee were obtained in 15 degrees of flexion using a fluoroscopically positioned x-ray beam. Axial view of the patellofemoral joint was acquired with vertical beam and the subject standing with the knee in 50 degrees of flexion [7].

The Ahlbäck classification used for OA grading is based on reduction of joint space and the attenuation of subchondral bone. The classification includes 5 grades of radiographic knee OA; grade 1: joint space narrowing $(<3 \mathrm{~mm})$, grade 2: joint space obliteration, grade 3: minor bone attrition $(<5 \mathrm{~mm})$, grade 4 : moderate bone attrition $(5-10$ $\mathrm{mm}$ ) and grade 5: severe bone attrition $(>10 \mathrm{~mm})$ [8]. The radiographs were classified by one orthopaedic surgeon (STL). The Ahlbäck grade 1 corresponds approximately to Kellgren \& Lawrence (K\&L) [9] OA grade 2-3 (minimal to moderate, definite osteophyte, unimpaired joint space to moderate dimunition of joint space) and the Ahlbäck grade grade 2-5 to K\&L OA grade 4 (severe, joint space greatly impaired with sclerosis of subchondral bone).

The preoperative knee alignment was assessed by the HKA angle. The HKA angle was obtained with the patient standing in a weight bearing position when radiographic anteroposterior and lateral views of the lower limb (hip, knee and foot) were taken. By drawing a line from the center of the femoral head to the midpoint of the tibial eminential spine and another line from this midpoint to the center of the talus surface of the ankle joint, the mechanical axis of the limb can be calculated [10]. The medial angle between the lines is the HKA angle (varus < $180^{\circ}$ ) (Figure 1). The accuracy and reproducibility of measurement of the HKA angle has been shown to be within 2 degrees [11]. In non-OA knees the mean HKA angle is 0.9-1.6 degrees in varus [12-14]. The HKA-angle was measured preoperatively as a part of the indication for surgery and postoperatively during the correction period to determine the progress of the correction and to determine that the desired alignment was obtained. The goal of correction is $4^{\circ}$ valgus for the varus knee. Taking the reproducibility of HKA-angle measurement into account, 2 degrees is accepted as optimal correction. All patients were radiographically examined at the same radiographic department, the radiographs were taken by experienced technicians and the HKA angle was determined by radiologists with expertise in musculoskeletal radiology.

\section{Pain}

Pain was measured by the subscale pain of the Knee injury and Osteoarthritisis Outcome Score (KOOS) preoperatively and at the 1 year follow-up [15]. KOOS is a 42 -item self-administrated knee-specific questionnaire based on the WOMAC index [16]. KOOS was developed to be used 


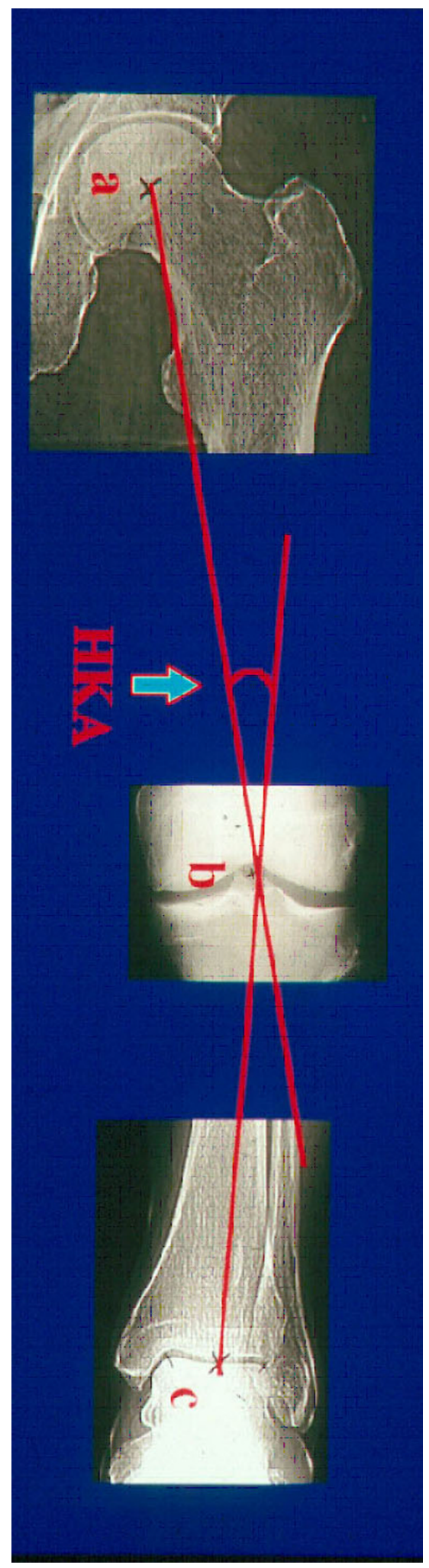

Figure I

Radiographic measurement of the Hip-Knee-Ankle angle (HKA-angle). for short-term and long-term follow-up studies of knee injury and knee OA. The KOOS comprises five subscales: pain, symptoms, activities of daily living function (ADL), sport and recreation function (Sport/Rec) and knee related quality of life (QOL). Standardized answer options are given (5 Likert boxes), and each respond is scored from 0 to 4 . A percentage score from 0 to 100 is calculated for each subscale; 100 representing the best possible results. 8-10 points of the KOOS score is considered a clinically relevant difference [17]. The KOOS is previously used in HTO [18].

Tibial osteotomy by the hemicallotasis technique (HCO) HCO is an open wedge osteotomy based on successive correction of the malalignment using an external fixation $[18,19]$ (Figure 2).

\section{Statistics}

The association between preoperative knee alignment (HKA angle) and preoperative knee pain (KOOS subscale pain), and change in knee alignment with surgery (the difference between preoperative HKA angle and postoperative HKA angle) and change in knee pain over time was assessed by simple regression analyses. Multiple regres-

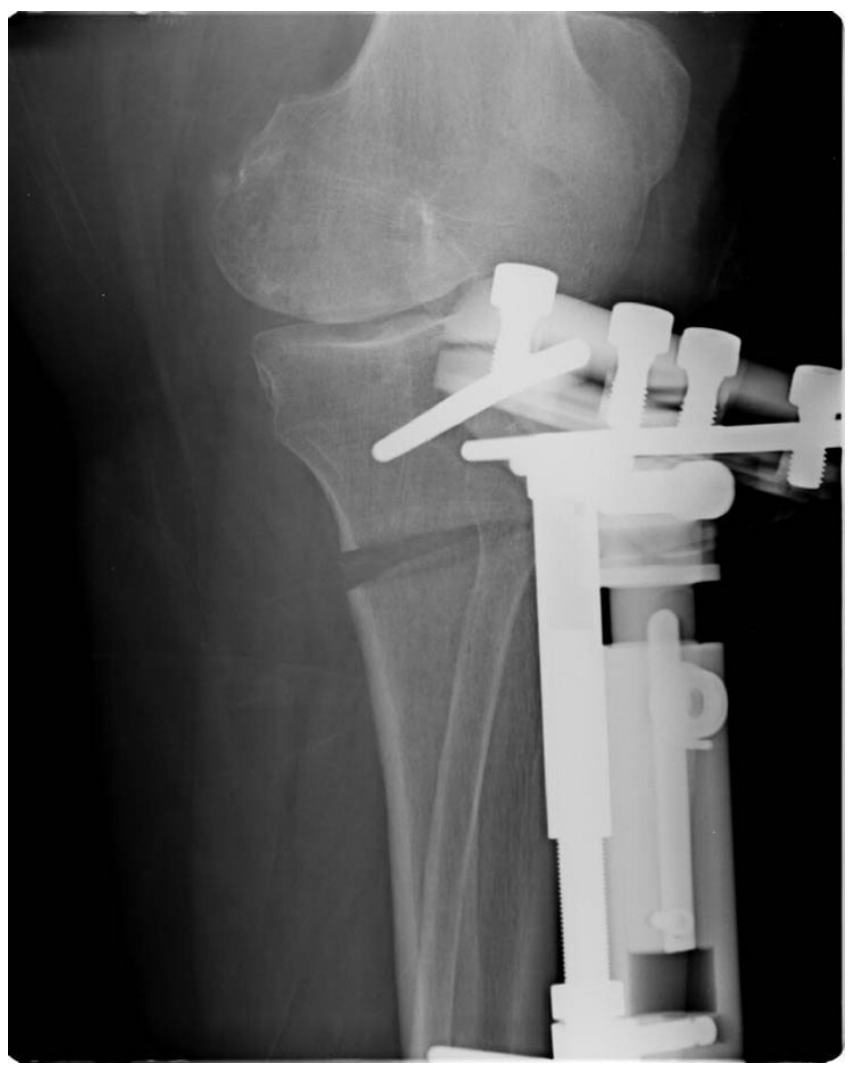

Figure 2

Radiograph of high tibial osteotomy using the hemicallotasis technique. 
sion analyses were used to control for potential confounding variables on preoperative KOOS pain (sex, age, Body Mass Index (BMI kg/m²), severity of knee OA (Ahlbäck grade 1-5) and preoperative knee alignment (HKA angle)) and on change in KOOS pain from preoperatively to the one year follow-up (sex, age, BMI, complications [septic arthritis, infection of the incision, DVT, replacement of pins, loss of correction and delayed healing], preoperative KOOS pain and change in knee alignment). The Ahlbäck grade 1 was used as reference and analyzed to Ahlbäck grade 2 and Ahlbäck grade $\geq 3$ respectively (the category Ahlbäck grade $\geq 3$ includes 13 patients with Ahlbäck grade 4 and one with Ahlbäck grade 5).

The results were presented with 95\% confidence intervals (95\% CI). P value $<0.05$ was considered as statistically significant.

The study was approved by the Ethics Committee at the Medical Faculty, Lund University (LU-565-1) and was performed in accordance with the Declaration of Helsinki.

\section{Results}

Patient characteristics for the 182 consecutive patients (mean age 52.8, 68\% men) available at baseline and the 156 patients available at the one year follow up are given in table 1.

\section{Preoperative cross-sectional analysis}

Preoperatively, the mean HKA-angle was 170 degrees, i.e. on average the patients had $10^{\circ}$ of varus alignment and the preoperative KOOS pain score was 42, (Table 1). There was no association between preoperative varus alignment and preoperative KOOS pain either crude or adjusted (Table 2).

\section{Longitudinal analysis}

156 patients $(86 \%)$ were available at the one year follow up (Table 1). The preferred correction (4 degrees valgus + / - 2 degrees) was obtained in 178/182 patients. The mean postoperative alignment was 184 degrees (range 171 $185)$. The mean change in knee HKA-angle was 13 degrees (range 0 - 30). The mean change in KOOS pain was 32 points (range -16 - 83). There was no association between change in knee alignment with surgery and change in knee pain preoperatively to one year postoperatively either crude or adjusted (Table 2).

Preoperatively, higher BMI and female gender were associated with more pain.

More preoperative pain predicted less improvement in pain postoperatively and patients with Ahlbäck OA grade 2 tended to have less improvement in KOOS pain over time than patients with Ahlbäck OA grade 1 and 3 (Table 2).

Increasing OA grade was associated with more varus alignment. There was a statistically significant difference between the Ahlbäck categories of knee OA severity and preoperative HKA-angle (Figure 3a). However there was no association between Ahlbäck categories of knee OA severity and pain (Figure $3 b$ ).

\section{Discussion}

We found no association between knee alignment and knee pain, neither preoperatively nor from preoperatively to one year postoperatively in patients operated on for medial knee OA by high tibial osteotomy using the hemicallotasis technique.

To our knowledge the association of alignment and pain has not previously been assessed in patients undergoing an intervention improving malalignment. The rationale for analysing this association is the belief that higher grade of preoperative HKA-angle may be related to less improvement in pain. However our results indicate that patients with more severe varus alignment experience similar pain relief from high tibial osteotomy by the hemicallotasis technique as patients with less varus alignment.

A strength of our study is the wide range of HKA angle and KOOS pain both preoperatively and over time. If there were any associations between preoperative HKA-angle and preoperative pain, or between change in pain and change in HKA-angle, the study had the possibility to detect them.

We used the Ahlbäck classification [8] to determine OA severity. The Ahlbäck classification, used especially in orthopedics and in northern Europe, primarily focus on reduction of the joint space as an indirect sign of cartilage loss while the more commonly used classification according to Kellgren \& Lawrence takes osteophytes, joint space narrowing or both into account [9]. The Ahlbäck system differentiates between more severe grades of OA than the classification of Kellgren \& Lawrence, which is useful in orthopedics and decisions relating to surgical treatment. The agreement between K\&L grade 2-3 and Ahlbäck grade 1 as well as K\&L grades 3-4 versus Ahlbäck grades 1-2 has been shown to be good (k 0.76 and 0.78) [20].

Our results differ from previous reported results on the relation of knee alignment and pain measuring alignment from long limb radiographs [1-3]. However our results are in line with results from studies measuring alignment from anteroposterior (AP) radiographs of the knee joint $[4,5]$. Reasons for the difference in results between studies 
Table I: Patient characteristics of the study group

\begin{tabular}{|c|c|c|c|c|c|c|}
\hline & \multicolumn{3}{|c|}{ Preoperatively } & \multicolumn{3}{|c|}{ I year postoperatively } \\
\hline & All & Men & Women & All & Men & Women \\
\hline & $\mathrm{n}=182$ & $n=123$ & $n=59$ & $\mathrm{n}=156$ & $\mathrm{n}=103$ & $\mathrm{n}=53$ \\
\hline \multicolumn{7}{|l|}{ Age year } \\
\hline mean & 52.8 & 53.7 & 51 & 53.2 & 54.1 & 51.5 \\
\hline range & $34-69$ & $36-69$ & $34-63$ & $35-69$ & $36-69$ & $35-63$ \\
\hline \multicolumn{7}{|l|}{ BMI kg/m2 } \\
\hline Mean & 28.9 & 28.8 & 29.3 & 29 & 28.7 & 29.6 \\
\hline Range & 17.9-39.7 & $23-39$ & 17.9-39.7 & $21-39.7$ & $23-39$ & $21-39.7$ \\
\hline \multicolumn{7}{|c|}{ Ahlbäck OA grade *(n)\# } \\
\hline OA grade I & 30 & 18 & 12 & 26 & 16 & 10 \\
\hline OA grade 2 & 60 & 40 & 20 & 50 & 32 & 18 \\
\hline OA grade 3 & 71 & 50 & 21 & 66 & 45 & 21 \\
\hline OA grade 4 & 13 & 9 & 4 & 9 & 7 & 2 \\
\hline OA grade 5 & 5 & I & 0 & I & I & 0 \\
\hline \multicolumn{7}{|c|}{ Preop HKA-angle } \\
\hline \multicolumn{7}{|l|}{ Degrees } \\
\hline Mean & 170.4 & 169.9 & 171.3 & 170.5 & 169.9 & 171.7 \\
\hline range & $153-178$ & $153-178$ & $159-178$ & $157-178$ & $157-178$ & $161-178$ \\
\hline \multicolumn{7}{|l|}{ KOOS Pain } \\
\hline Mean & 42 & 47 & 38 & 42 & 45 & 37 \\
\hline Range & $3-86$ & $3-86$ & $3-67$ & $3-81$ & $3-81$ & $3-67$ \\
\hline
\end{tabular}

*Ahlbäck classification [8]

\# missing data 7 patients

$\mathrm{BMI}=$ Body Mass Index

Pre op HKA angle = preoperativ HKA-angle

$(<180$ degree $=$ varus $)$

may include the different populations, different methodologies for assessment of alignment and pain and interpretation of data.

\section{Different populations}

In our study subjects about to have surgery for advanced OA were included which is in contrast to subjects recruited from the community with less advanced OA or at risk for knee OA [1-5]. However different study populations alone may not explain the difference as different methods were used.

\section{Assessment and interpretation of alignment}

Different methods as well as different axis are used to determine the degree of deformity of the lower extremity. The mechanical axis by full-limb radiographic measures, 
Table 2: Relation of independent variables on preoperative pain and change in pain preoperatively to the one year follow-up

\begin{tabular}{|c|c|c|c|c|c|c|}
\hline & \multicolumn{3}{|c|}{ Preoperative Pain } & \multicolumn{3}{|c|}{ Change in Pain } \\
\hline & $\Delta^{*}$ & $95 \% \mathrm{Cl}$ & $\mathrm{p}$-value & $\Delta^{* *}$ & $95 \% \mathrm{Cl}$ & $\mathrm{p}$-value \\
\hline Gender & -6.3 & $-12.6-0.1$ & 0.06 & -1.2 & $-9.6-7.1$ & 0.8 \\
\hline Age & -0.7 & $-0.5-0.4$ & 0.8 & 0.2 & $-0.4-0.8$ & 0.5 \\
\hline $\mathrm{BMI} \mathrm{kg} / \mathrm{m}^{2}$ & $-1 . I$ & $-1.9--0.2$ & 0.01 & -0.8 & $-1.9-0.2$ & 0.1 \\
\hline \multicolumn{7}{|l|}{ OA grade\# } \\
\hline grade 2 & 0.6 & $-7.9-9.2$ & 0.9 & -10.4 & $-21.5-0.7$ & 0.07 \\
\hline grade $\geq 3$ & 1.9 & $-6.6-10.4$ & 0.7 & -6.4 & $-17.2-4.3$ & 0.2 \\
\hline Preoperative HKA-angle & 0.3 & $-0.4-1$ & 0.4 & & & \\
\hline Change in HKA-angle \#\# & & & & 0.4 & $-0.6-1.4$ & 0.4 \\
\hline Complications & & & & -1.4 & $-10.4-7.6$ & 0.8 \\
\hline Preoperative Pain & & & & -0.5 & $-0.7--0.3$ & $<0.0001$ \\
\hline
\end{tabular}

* Estimated change of preoperative pain per unit change independent variable

** Estimated change of change in pain (preoperatively to the one year follow-up) per unit change of independent variable

\# OA grade according to the Ahlbäck (Ahl) classification, reference Ahl grade I

\#\# preoperative minus postoperative HKA-angle

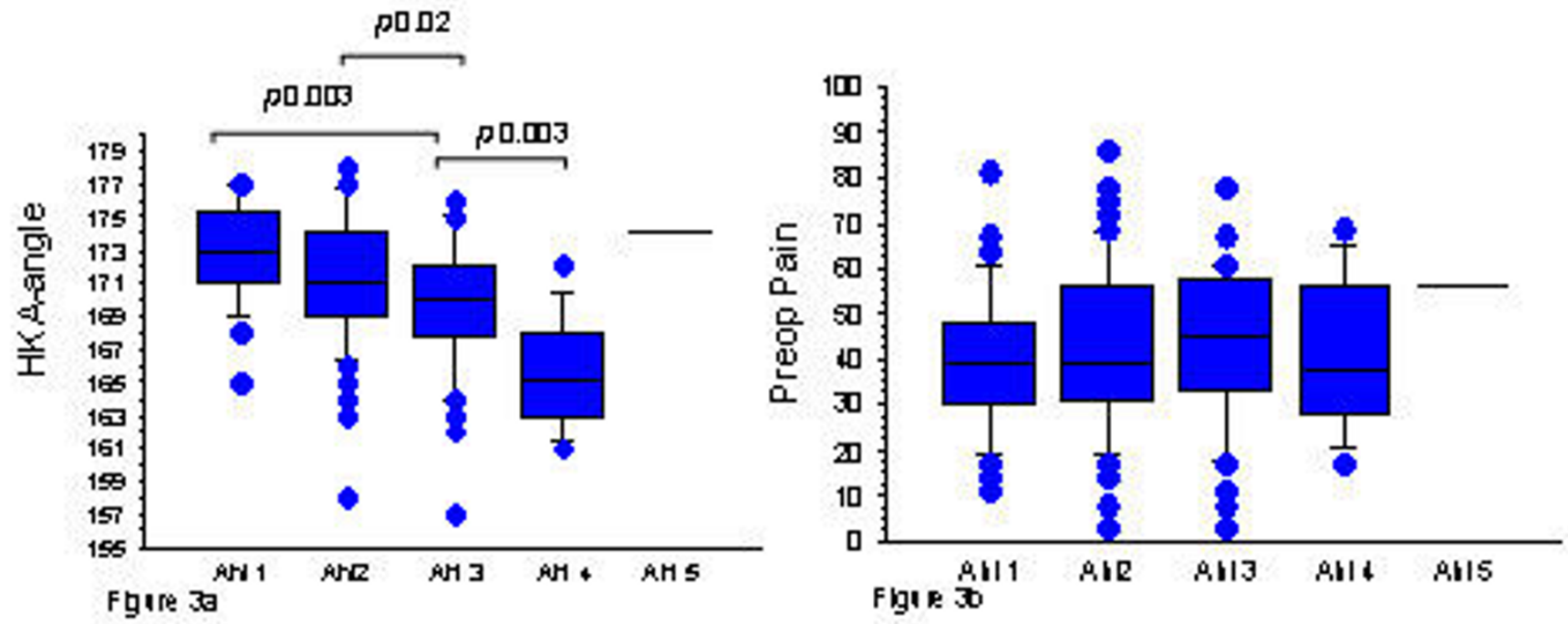

Figure 3

Boxplot of preoperative HKA angle (a) and preoperative pain (b) for each Ahlbäck grade of knee OA (Median with quartiles). Any data observation which lays more than I.5 IQR lower than the first quartile or higher than the third quartile is considered an outlier and marked as a dot. The horizontal line or "whisker" indicate where the smallest/highest value that is not an outlier by connecting it to the box). 
the HKA-angle, is used in association with surgical interventions such as high tibial osteotomy and knee replacement. Knee alignment is sometimes determined from anteroposterior (AP) radiographs of the knee joint. This measure is however uncertain because the shorter images includes limited parts of the femur and tibia and makes it impossible to determine neither mechanical nor anatomical axis of the lower extremity.

Measurement of different angles, using AP and long leg radiographs respectively, the error in the measurement, and different definitions of normal, varus and valgus alignment may explain the contradictory results. Studies analysing the association of knee alignment to knee pain has not reported or discussed the possible error in the measurement of neither the anatomical axis nor the mechanical axis $[2,4,5,21-23]$. The technique, experience and accuracy of the performance of the radiographic examination are of importance to minimize the methodological error. Aspects that makes the measurement of alignment of the lower leg uncertain.

\section{Assessment and interpretation of pain}

The mean KOOS pain score of 42 in this study is comparable to a preoperative score of 38 seen in patients having total knee replacement [24], indicating patients undergoing high tibial osteotomy having severe pain preoperatively. The mean improvement from high tibial osteotomy was 32 points at one year compared to 45 at one year after total knee replacement [24], indicating the effect of high tibial osteotomy being nearly as large as that from total knee replacement.

In previous studies the WOMAC $[4,5]$ and the Visual Analogue Scale (VAS) [2] have been used as pain measures. Different pain instruments may be of minor importance as long as valid instruments are used and instrument-specific clinically relevant differences are considered. Sharma et al (2001) showed for example differences of 3.5 - 16 $\mathrm{mm}$ in pain assessed by the VAS between three different categories of varus alignment and an average VAS increase of $10 \mathrm{~mm}$ on a $0-100 \mathrm{~mm}$ scale in knee pain with each $5^{\circ}$ of increased malalignment [2]. Clinically meningful differences in the Visual Analogue Scale (VAS) have been suggested to be $13-28 \mathrm{~mm}$ on a $100 \mathrm{~mm}$ scale depending on the initial VAS score [25].

In our study patients reported on average 1.5 KOOS points more pain on a 0-100 point scale per 5 degrees of varus alignment (Table 2). For the KOOS, an 8-10 point difference is considered a clinically relevant difference [17]. None of these studies showed clinically relevant differences with 5 degrees increasing malalignment, but the results were interpreted in opposite directions. Conclu- sions based on statistically significant results on the association of alignment to pain should be interpreted with caution if they are not clinically relevant.

In the cross sectional analysis preoperative pain was associated with increasing BMI while in the longitudinal analysis, there was no association. In the cross sectional analysis the change of preoperative pain per unit change of BMI was however negligible despite being significant. Patients with Ahlbäck grade 2 experienced clinically significant less improvement in pain over time compared to patients with Ahlbäck grade 1 but there was not a similar association for patients with Ahlbäck grade $\geq 3$. This may reflect the well-known discordance between radiographic knee OA and symptoms [26].

\section{Conclusion}

We found no association between knee alignment and knee pain in patients with knee OA indicating that alignment and pain are separate entities, and that the degree of preoperative malalignment is not a predictor of knee pain after surgery.

\section{Competing interests}

The authors declare that they have no competing interests.

\section{Authors' contributions}

AWD: study design, data selection, data analysis and preparation of manuscript. STL: data selection and preparation of manuscript. ER: study design, data analysis and preparation of manuscript. All authors read and approved the final manuscript.

\section{Acknowledgements}

We thank the Department of Radiology, Lund University Hospital for their contribution to the study. We also thank Jonas Ranstam, PhD, Statistician, National Musculoskeletal Competence Centre, Department of Orthopaedics, Lund University Hospital for valuable statistical advice.

This study was supported by Region Skåne. ER was supported by the Swedish Research Council, Region Skåne, and the Faculty of Medicine, Lund University.

\section{References}

I. Kraus VB, McDaniel G, Worrell TW, Feng S, Vail TP, Varju G, Coleman RE: Association of bone scintigraphic abnormalities with knee malalignment and pain. Ann Rheum Dis 2009, 68:1673-1679.

2. Sharma L, Song J, Felson DT, Cahue S, Shamiyeh E, Dunlop DD: The role of knee alignment in disease progression and functional decline in knee osteoarthritis. Jama 200I, 286: I88-95.

3. Sharma L, Felson DT, McCulloch C, Dunlop D, Hietpas J, Torner J, Lewis CE, Segal N, Curtis J, Guermazi A, Xie H, Niu J, Nevitt M: Relationship of Varus-Valgus Alignment to Incident Knee Symptoms. American College of Rheumatology Annual Scientific Meeting: I215 November 2006; Washington DC.

4. Lim BW, Hinman RS, Wrigley TV, Bennell KL: Varus malalignment and its association with impairments and functional limita- 
tions in medial knee osteoarthritis. Arthritis Rheum 2008, 59:935-42.

5. Lim BW, Hinman RS, Wrigley TV, Sharma L, Bennell KL: Does knee malalignment mediate the effects of quadriceps strengthening on knee adduction moment, pain, and function in medial knee osteoarthritis? A randomized controlled trial. Arthritis Rheum 2008, 59:943-5I.

6. Maly MR, Costigan PA, Olney SJ: Mechanical factors relate to pain in knee osteoarthritis. Clin Biomech (Bristol, Avon) 2008, 23:796-805

7. Boegard T, Jonsson K: Radiography in osteoarthritis of the knee. Skeletal Radiol 1999, 28:605-15.

8. Ahlback S: Osteoarthrosis of the knee. A radiographic investigation. Acta Radiol Diagn 1968:7-72.

9. Kellgren J, Jeffrey M, Ball J: Atlas of standard radiographs Volume 2. Oxford: Blackwell Scientific; 1963.

10. Siu D, Cooke TD, Broekhoven LD, Lam M, Fisher B, Saunders G, Challis TW: A standardized technique for lower limb radiography. Practice, applications, and error analysis. Invest Radiol | 99|, 26:7|-7.

II. Odenbring S, Berggren AM, Peil L: Roentgenographic assessment of the hip-knee-ankle axis in medial gonarthrosis. A study of reproducibility. Clin Orthop Relat Res 1993, 289:195-6.

12. Edholm P, Lindahl O, Lindholm B, Myrnerts R, Olsson KE, Wennberg E: Knee instability. An orthoradiographic study. Acta Orthop Scand 1976, 47:658-63.

13. Hsu R, Himeno S, Coventry MB, Cahue EYS: Normal axial alignment of the lower extremity and load bearing capacity at the knee. Transactions of the 34th annual meeting of the orthopedic research saociety Park Ridge, IL 1988.

14. Moreland JR, Bassett LW, Hanker G]: Radiographic analysis of the axial alignment of the lower extremity. J Bone Joint Surg Am 1987, 69:745-9.

15. Roos EM, Roos HP, Lohmander LS, Ekdahl C, Beynnon BD: Knee Injury and Osteoarthritis Outcome Score (KOOS)--development of a self-administered outcome measure. J Orthop Sports Phys Ther 1998, 28:88-96.

16. Bellamy N, Buchanan WW, Goldsmith CH, Campbell J, Stitt LW: Validation study of WOMAC: a health status instrument for measuring clinically important patient relevant outcomes to antirheumatic drug therapy in patients with osteoarthritis of the hip or knee. J Rheumatol 1988, I 5: 1833-40.

17. Roos EM, Lohmander LS: The Knee injury and Osteoarthritis Outcome Score (KOOS): from joint injury to osteoarthritis. Health Qual Life Outcomes 2003, I:64.

18. W-Dahl A, Toksvig-Larsen S, Roos EM: A 2-year prospective study of patient-relevant outcomes in patients operated on for knee osteoarthritis with tibial osteotomy. BMC Musculoskelet Disord 2005, 6: 18

19. Magyar G, Toksvig-Larsen S, Lindstrand A: Open wedge tibial osteotomy by callus distraction in gonarthrosis. Operative technique and early results in 36 patients. Acta Orthop Scand 1998, 69:|47-5|.

20. Petersson IF, Boegard T, Saxne T, Silman AJ, Svensson B: Radiographic osteoarthritis of the knee classified by the Ahlback and Kellgren \& Lawrence systems for the tibiofemoral joint in people aged 35-54 years with chronic knee pain. Ann Rheum Dis 1997, 56:93-6.

21. Brouwer GM, van Tol AW, Bergink AP, Belo JN, Bernsen RM, Reijman M, Pols HA, Bierma-Zeinstra SM: Association between valgus and varus alignment and the development and progression of radiographic osteoarthritis of the knee. Arthritis Rheum 2007, 56: I 204-II.

22. Hunter DJ, Niu J, Felson DT, Harvey WF, Gross KD, McCree P, Aliabadi $P$, Sack B, Zhang Y: Knee alignment does not predict incident osteoarthritis: the Framingham osteoarthritis study. Arthritis Rheum 2007, 56:1212-8.

23. Kraus VB, Vail TP, Worrell T, McDaniel G: A comparative assessment of alignment angle of the knee by radiographic and physical examination methods. Arthritis Rheum 2005, 52: 1730-5.

24. Roos EM, Toksvig-Larsen S: Knee injury and Osteoarthritis Outcome Score (KOOS) - validation and comparison to the WOMAC in total knee replacement. Health Qual Life Outcomes 2003, I: 17 .

25. Bird $S B$, Dickson EW: Clinically significant changes in pain along the visual analog scale. Ann Emerg Med 200I, 38:639-43.
26. Hannan MT, Felson DT, Pincus T: Analysis of the discordance between radiographic changes and knee pain in osteoarthritis of the knee. J Rheumatol 2000, 27:1513-7.

\section{Pre-publication history}

The pre-publication history for this paper can be accessed here:

http://www.biomedcentral.com/1471-2474/10/154/pre pub

Publish with Biomed Central and every scientist can read your work free of charge

"BioMed Central will be the most significant development for disseminating the results of biomedical research in our lifetime. "

Sir Paul Nurse, Cancer Research UK

Your research papers will be:

- available free of charge to the entire biomedical community

- peer reviewed and published immediately upon acceptance

- cited in PubMed and archived on PubMed Central

- yours - you keep the copyright 